\title{
Review of current research in the prevention of nerve damage in leprosy
}

\author{
W. C. S. SMITH \\ Department of Public Health, University of Aberdeen Medical School, \\ Aberdeen, UK
}

Summary Nerve damage and the consequences of nerve damage set leprosy apart from other diseases. The irreversible motor and sensory impairments caused by leprosy lead to increasing secondary impairments long after the disease process has been arrested. Interventions that prevent, reverse or limit the impairments resulting from leprosy are, therefore, of the highest priority. Self-care has been demonstrated to be an effective means of preventing secondary tissue damage, and its implementation must now be encouraged within the framework of basic health care. Currently, a comprehensive effort has been made to address all dimensions of impairment of nerve function and its consequences, from prevention of both primary and secondary impairments to interventions in long-term nerve damage. The BANDS prospective study of a cohort of 2664 previously untreated leprosy patients in Bangladesh has provided both a valuable baseline and insight into the epidemiology of nerve damage in leprosy. This work has defined the numbers of patients who present with acute nerve damage, the numbers who are not treated, and the importance of the MB classification, and has described the simple clinical prediction rule. This study also provided the basis for the development of the three TRIPOD randomized clinical trials in Nepal and Bangladesh. Follow-up of the BANDS cohort, which is continuing, will yield more data on the natural history of nerve damage. The encouraging results from the TRIPOD trials of low-dose prophylactic steroids will be followed up, to see whether the effect is sustained over time. The TRIPOD trials are to be completed, a trial of three steroid regimens in reversal reaction is to be completed, and a number of specific research topics within the ILEP programme, INFIR, are to be developed.

\section{Introduction}

Nerve damage and the consequences of nerve damage set leprosy apart from other diseases. ${ }^{1}$ The irreversible motor, sensory, and autonomic impairments caused by leprosy lead to increasing secondary impairments long after the disease-process has been arrested. The progressive physical impairments caused by the disease process are compounded by the psychological and social consequences that adversely influence the participation in society of those affected. Research into interventions that prevent, reverse or limit the impairments resulting from leprosy is therefore of the highest priority.

The magnitude of the problem has been estimated from the proportion of newly diagnosed patients who have WHO disability grade 2 at detection. ${ }^{2}$ However, this yields 
an underestimate of the size of the problem, as it does not include those with only sensory impairments, those who develop impairments after detection, and any of the social consequences. Moreover, this estimate does not include those who have completed a course of multidrug therapy (MDT), and are therefore no longer registered for treatment, but nevertheless have nerve function impairment (NFI) and the potential for progressive tissue damage as a result of motor, autonomic and sensory impairment. Estimates of the numbers affected globally vary upwards from 1.3 million, depending upon a number of assumptions about the age and life-expectancy of those affected. ${ }^{3}$ The numbers of newly discovered patients with grade 2 disability at detection have been decreasing globally, reflecting generally improved case detection and treatment. However, there is considerable country-to-country variation in the proportion of new cases presenting with WHO grade 2 disability, and a tendency for countries with fewer cases to have larger proportions. This tendency is a concern for the future, and the trend of the number of new cases with grade 2 disability must be monitored carefully. Nerve damage that has already developed by the time of diagnosis is important, because it may be prevented by early case-detection and treatment with MDT.

Current research represents a comprehensive effort to address all dimensions of NFI and its consequences, from prevention of both primary and secondary impairments to interventions in long-term nerve damage. These areas of research have been identified as a priority for research, ${ }^{4}$ and have been taken up by a number of member associations of the International Federation of Anti-Leprosy Associations (ILEP), which have committed funds to support these research initiatives. ${ }^{5}$

\section{Review of current research}

This review describes the development and current progress of four separate but linked programmes of research in the field of nerve damage. The work represents a progressive development, aimed at improving our understanding of the mechanisms of nerve damage, developing practical tools for preventing nerve damage, improving its treatment when it occurs, and minimizing the long term impact on individuals, their families and society.

The four studies are: (i) an observational cohort study of several thousand newly detected patients in Bangladesh (BANDS); (ii) a series of intervention trials-randomized, controlled trials in the prevention of disability in leprosy (TRIPOD), that employ steroids to prevent or reverse nerve damage; (iii) a multicentre trial in India of different steroid regimens for the treatment of type 1 (reversal) reactions; and (iv) a programme of collaborative research that addresses issues of nerve impairment and reactions (INFIR).

\section{BANDS (Bangladesh Acute Nerve Damage Study)}

BANDS is a prospective study of a cohort of 2664 previously untreated leprosy patients in Bangladesh, that has provided a valuable baseline and insight into the epidemiology of nerve damage in leprosy. ${ }^{6}$ This work has determined the numbers of patients who presented with acute nerve damage ${ }^{7}$ and the numbers not treated, demonstrated the importance of the MB classification, and described the 'simple clinical prediction rule'. ${ }^{8}$ The study provided the basis for the development of the three TRIPOD trials being conducted in Nepal and Bangladesh. The BANDS data provided estimates of the frequency of events that facilitated 
calculations of sample-size and planning the study design for those trials. The BANDS data have also proved of value in planning the programme of research on NFI and reactions in leprosy (INFIR) that is coordinated by ILEP.

\section{TRIPOD (Trials in the Prevention of Disability in Leprosy)}

The results of the BANDS study in Bangladesh were consistent with those of research on impairment of nerve function conducted earlier in Nepal. ${ }^{9}$ Three distinct questions relating to the detection and treatment of NFI were identified, and a programme of collaborative research was developed to address the questions: (i) could NFI and reactions occurring during MDT be prevented using prophylactic corticosteroids, as had been suggested by a pilot study ? $^{10}$ (ii) Would earlier detection of NFI, using more refined detection methods such as monofilaments, and earlier treatment lead to a better outcome? And (iii) would NFI of longer than 6 months duration respond to steroid therapy? Standard practice is not to treat NFI of longer than 6 months duration; however, recent work suggests that some recovery of function may be possible. ${ }^{11}$

The TRIPOD programme of research on the prevention of impairment and disability in leprosy was developed by a group of researchers in Bangladesh and Nepal, supported by staff at the University of Aberdeen, and financial support has been provided by LEPRA. The trials required the recruitment of a number of new centres in both Nepal and Bangladesh. The aim of the TRIPOD trials was to investigate the efficacy of corticosteroid treatment in prevention of impairment and disability in leprosy. There were three trials with the following specific objectives: (i) POD 1-to investigate whether corticosteroids administered in low dosage would reduce the frequency of NFI events among newly diagnosed MB leprosy patients; (ii) POD 2-to investigate whether patients who have normal sensation when tested with a ball-point pen, but who have sensory impairment when tested with monofilaments, would have a better outcome if treated with corticosteroids; and (iii) POD 3-to investigate whether patients who have untreated NFI, which commenced between 6 and 24 months previously, would benefit from corticosteroid treatment.

The detailed protocols, including detailed criteria for inclusion and exclusion, were developed and agreed upon by all of the investigators. Centres in each country were recruited, and staffs were trained in the study methods. Each POD trial employed a multicentre, randomized, double-blind, placebo-controlled design. The corticosteroids and placebo were produced in Bangladesh, and the randomization was personally supervised by two of the investigators. Independent assessors, from whom the nature of the treatment had been concealed, evaluated the outcomes at fixed end-points. Sample size calculations were based on estimates from previous research, and assumed a 50\% reduction of the frequency of NFI events, or, in POD 3,50\% improvement of NFI. The numbers of patients to be recruited into the trials were 770 in POD 1, 360 in POD 2, and 200 in POD 3.

Funding of the study began in June 1997, and the planned duration of the study was 30 months. An interim analysis of the results by the trial analyst was to take place when $50 \%$ of the patients had completed 4 months of follow-up, to assess safety and efficacy while preserving the double-blind character of the trials. This analysis, conducted in 1999, demonstrated that steroids can be used safely on a large scale if patients are carefully selected and monitored, and that the trial was progressing satisfactorily. Although the results of the 6-month follow-up are encouraging, the important outcome will be the benefits 
sustained over the long term. Recruitment of new patients ended in June 2000, and, after 4 months of steroid or placebo, the 12-month follow-up of the last patients recruited will be completed by the end of October 2001.

\section{Reversal reaction trial in India}

Many leprosy control programmes had implemented standard, fixed-dose and -duration regimens for treatment of reactions and acute NFI, ${ }^{13,14}$ primarily for use in field programmes, in which it was impossible to supervise treatment closely and vary treatment with steroids according to changes of nerve function. In some areas, steroids were packaged in 'calendar' or 'blister' packs, to facilitate administration in the field, particularly in those areas in which leprosy control activities are integrated within the primary healthcare services. However, the results of the BANDS study ${ }^{12}$ indicated, as had earlier studies, that not all patients with acute NFI are detected and treated promptly with steroids, and not all patients treated with steroids recover nerve function.

A randomized, double-blind, controlled trial in six centres in India, coordinated by the Schieffelin Leprosy Research and Training Centre, Karigiri, is examining the effect of steroid dosage and duration, in order to optimize the standard steroid regimen for the treatment of reversal reactions and acute nerve damage to primary-care settings.

\section{INFIR (ILEP Co-ordinated Programme of Research on Nerve Function Impairment and Reactions in Leprosy)}

In December 1997, the working session of the ILEP Medical and Social Commission identified nerve damage as an urgent research issue, and recommended that a workshop be convened to review the current state of research in reactions and nerve damage, and to identify the priorities for research. A workshop was held in London in June 1998, bringing together researchers representing a wide range of disciplines, who were nominated and supported by ILEP member associations. Stimulated by input from experts in other neurological diseases that share some pathogenetic mechanisms with the nerve damage of leprosy, the workshop critically reviewed questions relating to the prediction, detection, and treatment of reactions in leprosy.

Four major research themes were proposed as a result of the workshop, which together form a programme of collaborative research. The four projects that make up the programme of research are as follows.

(I) A COHORT STUDY TO INVESTIGATE THE PREDICTION, DETECTION AND PATHOGENESIS OF NERVE FUNCTION IMPAIRMENT IN UNTREATED, NEWLY DIAGNOSED LEPROSY PATIENTS

Leprosy is feared because of the deformities and disability that it may cause. From the point of view of the leprosy patient, successful treatment is that which prevents or heals impairments, deformities and disabilities, most of which result from NFI, often caused by immunological reactions against antigens of Mycobacterium leprae. In recent years, it has been recognized that people remain at risk of such immunologically mediated nerve damage even during and after successful anti-leprosy treatment. 
A number of measures have been proposed, that may be useful in predicting which patients would develop NFI and reactions. These must be tested in a carefully planned, prospective study that follows patients serially over a period of time. In addition to nerve conduction studies, a number of new methods of assessing nerve function are now available, including laser Doppler and thermal threshold testing; these must be evaluated, and compared to more routine methods, such as monofilament and ball-point pen testing.

Our understanding of the immunopathology of NFI and reactions is still very incomplete. Much of the current knowledge has been gained from cross-sectional studies. Therefore, there is a need for a longitudinal study in which the immunological and histopathological aspects are studied concurrently.

This cohort study, of 300 previously untreated, newly diagnosed MB patients, is designed to address all three aims simultaneously: to assess measures that may predict NFI; to assess methods of detecting NFI; and to study the pathogenesis of NFI and reactions in leprosy. The patients are to be treated with MDT and followed monthly for a minimum of 1 year. Pilotstudies are now being set up in two leprosy centres in Northern India, in collaboration with research laboratories in Miraj and Hyderabad, India.

\section{(II) TRIALS OF NEW TREATMENTS OF REACTIONS IN LEPROSY}

At present, the treatment of leprosy reactions is largely dependent on steroids, together with clofazimine and thalidomide. The results of treatment with steroids are not always satisfactory, and there are ongoing trials to develop optimal regimens. Also, treatment with steroids is not without adverse effects, which include diabetes, hypertension, tuberculosis and peptic ulceration. Two new treatment possibilities, cyclosporin A and azathioprine, that may be valuable in the management of reactions, have not yet been tested. As yet, there is no evidence of their effectiveness in reactions, and phase I and phase II trials must be conducted. If these are successful, additional, randomized trials may be conducted. However, even if these drugs do not become standard for treatment of reactions in field conditions, demonstration of their efficacy would provide important understanding of the mechanisms of reactions in leprosy. The aims of these studies are to measure the efficacy of new treatments, including cyclosporin A and azathioprine, of reactions. The initial work is based on small numbers of patients, employing open trial designs. The numbers of patients, dosages of drugs, and other details are currently being developed in collaboration with field centres.

\section{(III) RECURRENT AND LATE REACTIONS IN LEPROSY}

Much of the research into the patterns and predictors of reactions has focused on the time to first episode, or on a comparison of those who have experienced a reaction with those who never experience a reaction. However, recurrent reactions, which are of great importance in the field, because they are difficult to treat, have been a concern for many treatment studies. Can we identify which patients will develop recurrent reactions? One data set that has been analysed showed that, whereas only $5 \%$ of patients experienced reversal reaction, $60 \%$ of those who experienced one episode had a second episode, and $60 \%$ of those who experienced a second episode had a third.

Late reactions are important in the field, and the staffs of leprosy control programmes need to be able to predict who will develop late reactions. Also, as the duration of chemotherapy is shortened, we need to know what proportions of patients develop the first 
reaction after 6, 12 or 24 months. These questions are important for the effective management of field programmes, and for determining policies for long-term follow-up and surveillance.

The aim of the study is to describe the factors that predict recurrent reactions, and to predict which patients will develop reactions after 12 and 24 months. Many data sets exist that could provide the information needed to answer these questions. The design of this study involves the adoption of a standard method of analysis, its application to the existing data sets individually, and production of a meta-analysis of the results from all the centres. The principal investigators from each centre are to participate in a workshop to be held in November 2000, to present the data, and to discuss their interpretation prior to publication as a series of articles and a single review of all the studies.

(IV) DELAY OF PRESENTATION AND START OF TREATMENT OF PATIENTS WITH LEPROSY

Early detection and treatment of leprosy can prevent damage to the peripheral nerves. However, a large number of patients already have irreversible NFI when they present to the health services. Studies aimed at identifying programme and patient reasons why some patients present late are relevant, as they can provide programme managers with the tools with which to promote early reporting and, consequently, the start of appropriate treatment.

This proposal aims at developing standard quantitative and qualitative methods of identifying the factors involved in delayed presentation and start of treatment of leprosy patients that can be used in different cultural settings. The study will be carried out in different countries in different socio-cultural settings, with the purpose of identifying the socio-cultural, health service and patient factors associated with delay of presentation and start of treatment of leprosy patients, and, on the basis of the findings, to recommend means by which to investigate the causes of the delays and reduce them.

Initially, quantitative data will be collected, to measure the duration of the delay. Patient record cards will be the source of information, and the disability grade at diagnosis will be used as an indicator. The second phase, which will be qualitative, will aim to answer the question of why a delay occurs, and which factors contribute to it. The needed information will be collected from the community as well as from the leprosy patients, and will include data related to the health services and the quality of care provided. Research tools will include questionnaires and checklists for focus-group discussions, checklists for observation of health workers, and in-depth interviews with patients, staff and community leaders.

\section{Conclusions}

The programmes of research described above will improve our understanding of the epidemiology of reactions and nerve damage in leprosy. And, because the research progresses from observational studies to trials of interventions, it may also improve current methods of prevention and treatment of nerve damage in leprosy.

A major priority for the future is to apply the findings of the current work on prevention of NFI to field programmes, particularly those based in primary health-care settings. Self-care, which has been demonstrated to be an effective means of preventing secondary tissue damage, ${ }^{15}$ must now be adapted for implementation within basic health care. The future of all leprosy programmes, both in leprosy control and in rehabilitation, will be in integrated settings. It is therefore essential that innovation in the area of prevention of nerve damage in 
leprosy be carried out in such integrated environments. The research addresses the major priority area of nerve damage that is critically important for patients and their families, and will have a significant impact on reducing the burden of disease.

\section{Acknowledgements}

The research programmes described in this review represent the work of several teams of people, working across disciplines and in collaboration from many institutions and many countries. The investigators are, in the BANDS study, R. Croft, J. H. Richardus, P. Nicholls and W. C. S. Smith, and, in the TRIPOD trials, A. Anderson, R. Croft, J. H. Richardus, S. Withington, W. van Brakel, P. Nicholls and W. C. S. Smith. The reversal reaction trial in India is co-ordinated by P. S. S. Sunda Rao, B. P. Ravi Kumar and T. Sugumaran. The steering group for the INFIR Programme includes M. J. Colston, D. Lockwood, P. Nicholls, W. van Brakel, W. C. S. Smith, E. Declerq, W. Britton and D. Soutar. ILEP Member Associations including LEPRA, TLMI, ALM, and Luxembourg Follereau Foundation fund most of the work described.

\section{References}

${ }^{1}$ Smith WCS, Parkhe SM. Disability assessment as a measure of progress in leprosy control. Lepr Rev, 1986; 57: 251-259.

2 World Health Organization. Global case-detection trends in leprosy. Weekly Epidemiol Rec, 1997; 72: 173-180.

${ }^{3}$ World Health Organization. Leprosy disabilities: magnitude of the problem. Weekly Epidemiol Rec, $1995 ;$ 70: 269-276.

${ }^{4}$ Smith WC, van Brakel WH. Bangkok Workshop on Leprosy Research. Research needs related to disabilities and rehabilitation. Int J Lepr, 1996; 64: S52-54.

5 Martineau-Needham D, Lacey S. Leprosy control activities of the International Federation of Anti-leprosy Associations. World Health Statistics Quarterly, 1991; 44: 36-46.

${ }^{6}$ Croft RP, Richardus JH, Nicholls PG, Smith WC. Nerve function impairment in leprosy: design, methodology, and intake status of a prospective cohort study of 2664 new leprosy cases in Bangladesh (The Bangladesh Acute Nerve damage Study). Lepr Rev, 1999; 70: 140-159.

7 Croft RP, Nicholls PG, Richardus JH, Smith WC. Incidence rates of acute nerve function impairment in leprosy: a prospective cohort analysis after 24 months (The Bangladesh Acute Nerve damage Study). Lepr Rev, 2000; 71: $18-33$.

8 Croft RP, Nicholls PG, Steyerberg EW et al. A clinical prediction rule for nerve function impairment in leprosy patients. Lancet, 2000; 355: 1603-1606.

9 Van Brakel WH, Khawas IB. Nerve damage in leprosy: an epidemiological and clinical study of 396 patients in west Nepal_-part 1. Definitions, methods and frequencies. Lepr Rev, 1994; 65: 204-221.

10 Croft RP, Nicholls P, Anderson AM et al. Effect of prophylactic corticosteroids on the incidence of reactions in newly diagnosed multibacillary leprosy patients [letter]. Int J Lepr, 1999; 67: 75-77.

11 Croft R, Richardus JH, Smith WCS. The effectiveness of corticosteroids in the treatment of long-term nerve function impairment. Lepr Rev, 1996; 67: 342-343.

12 Croft RP, Richardus RH, Smith WC. Field treatment of acute nerve function impairment in leprosy using a standardized corticosteroid regimen-first year's experience with 100 patients. Lepr Rev, 1997; 68: 316-325.

13 Smith WC. Prevention of disability in leprosy-ILEP medical bulletin. Lepr Rev, 1996; 67: 68-72.

14 World Health Organization. A guide to eliminating leprosy as a public health problem. Geneva 1997; WHO/LEP/ 97.7.

15 Smith WC, Zhang G, Zheng T, Watson JM, Lehman LF, Lever P. Prevention of impairment in leprosy; results from a collaborative project in China. Int J Lepr, 1995; 63: 507-517. 


\section{DISCUSSION}

Professor Nath: In India, we see patients who have neural involvement without skin lesions. No one has commented on these patients. Is this because they are not seen outside India?

Professor Smith: The BANDS database contains a number of such patients; altogether, they are a small group.

Dr Modlin: From $20 \mathrm{mg}$ prednisolone daily for a protracted period, would you expect side-effects to be sufficiently obvious so that the study would no longer be 'blind'?

Professor Smith: Very little difference of side-effects was reported. Two patients were removed from the study because of adverse reactions that were thought to be caused by steroids, but neither patient had been treated with steroids. In fact, the patients were screened at entry for respiratory symptoms suggestive of pulmonary tuberculosis, and for hypertension, so as to exclude those who might be most likely to suffer adverse reactions from the steroids. And it is because of our concerns for side-effects that an interim analysis was planned. In fact, the interim analysis, performed by an independent observer, showed no evidence of adverse reactions to the steroids.

Professor Ji: Have you noted any difference of the rate of decline of the BI between those treated with steroids and those not so treated?

Professor Smith: We haven't examined this point. 